\title{
Magneto-Ionic Propagation in Inhomogeneous Media. Part II. Oblique Propagation
}

\author{
B. K. Banerjea \\ Institute of Ārmament Technology, Poona, India
}

(Received March 29, 1965)

\begin{abstract}
Oblique propagation of electromagnetic waves in an inhomogeneous magneto-ionic medium is described by a pair of coupled, linear, second-order differential equations, with coefficient functions having one essential singularity and more than one pole. An analytic method to study this general type of propagation has been developed in this paper.

Mathematical expressions for all the physical observables in the ionospheric investigations have been derived for a parabolic electron layer. The expressions for the phase retardation and the tilt of the polarization ellipse differ radically from those derived from the ray optics of Appleton and Hartree.
\end{abstract}

\section{Wave Equations}

The general type of magneto-ionic propagation is described by the equation

$$
\left[\frac{\partial}{\partial t}+\nu+\omega_{H} \times\right]\left[\boldsymbol{\nabla} \times \boldsymbol{\nabla} \times \boldsymbol{E}+\mu_{0} \epsilon_{0} \frac{\partial^{2} \boldsymbol{E}}{\partial t^{2}}\right]+\frac{\mu_{0} N e^{2}}{m} \frac{\partial \boldsymbol{E}}{\partial t}=0
$$

where mks units and symbols (see appendix) have been used.

The statement of the physical problem and a brief summary of the previous work in this line have been discussed in part I of this paper.

In the above wave equation let, (i) the partial time derivatives be replaced by $i \omega$; (ii) the steady magnetic field $\boldsymbol{H}_{e},\left(\omega_{H}=e \mu_{0} \boldsymbol{H}_{e} / m\right)$ be in the $x z$-plane with the vertical $z$-axis as the direction of propagation; (iii) the collision frequency $\nu$ be independent of $z$; and (iv) $N$, the electron density, be given by

$$
\begin{aligned}
& N(z)=N_{m}\left(1-\frac{z^{2}}{l^{2}}\right) \text { for } z^{2}<l^{2}, \\
& =0 \quad \text { for } z^{2}>0
\end{aligned}
$$

where $z=0$ is fixed at the layer of maximum ionization.

Under the above set of assumptions (1) decomposes into

$$
\frac{d^{2} E_{x}}{d z^{2}}+\left[K_{1}(z) E_{x}-i Q \mathrm{~L}(z) E y\right]=0, \quad \frac{d^{2} E y}{d z^{2}}+\left[K_{2}(z) E_{y}+i Q \mathrm{~L}(z) E_{x}\right]=0,
$$

where

$$
\begin{aligned}
K_{1}(z) & =K^{2} \frac{\left(z^{2}-a_{1}^{2}\right)\left(z^{2}-b_{1}^{2}\right)}{\left(z^{2}-z_{0}^{2}\right)}, \quad K_{2}(z)=K^{2} \frac{\left(z^{2}-a_{2}^{2}\right)\left(z^{2}-b_{2}^{2}\right)}{\left(z^{2}-z_{0}^{2}\right)}, \quad L(z)=K^{2} \frac{\left(z^{2}-a_{1}^{2}\right)\left(z^{2}-l^{2}\right)}{\left(z^{2}-z_{0}^{2}\right)} \\
Q & =h_{3} \cos \theta / h_{2}, \quad K^{2}=4 \pi h_{1}^{2} /(1-Q) l^{4}, \quad \omega_{m}^{2}=\frac{N_{m} e^{2}}{m \epsilon_{0}}=\frac{4 \pi^{2} c^{2}}{\lambda_{m}^{2}}, \\
h_{1}^{2} & =l^{2} /(1-i Z) \lambda_{m}^{2}, \quad h_{2}^{2}=(1-i Z) \omega^{2} / \omega_{m}^{2}, \quad h_{3}^{2}=\omega_{H}^{2} /(1-i Z) \omega_{m}^{2},
\end{aligned}
$$




$$
\begin{aligned}
& Z=1-i \nu / \omega, \quad \theta=\frac{\pi}{2}+\delta, \quad \delta=\text { magnetic dip angle, } \\
& a_{1}^{2}=\left(1-h_{2}^{2}\right) l^{2}, \quad a_{2}^{2}=\left[1-h_{2}^{2}+\frac{h_{3}^{2} \cos ^{2} \theta}{2}+h_{2} h_{3} \sqrt{\sin ^{2} \theta+\frac{Q^{2} \cos ^{2} \theta}{4}}\right] l^{2}, \\
& b_{1}^{2}=\left(1-h_{2}^{2}+h_{3}^{2}\right) l^{2}, \quad b_{2}^{2}=\left[1-h_{2}^{2}+\frac{h_{3}^{2} \cos ^{2} \theta}{2}-h_{2} h_{3} \sqrt{\sin ^{2} \theta+\frac{Q^{2} \cos ^{2} \theta}{4}}\right] l^{2}, \\
& z_{0}^{2}=\left[1-h_{2}^{2}+\frac{h_{3}^{2} \sin ^{2} \theta}{\left(1-Q^{2}\right)}\right] l^{2} .
\end{aligned}
$$

For $\theta=\frac{\pi}{2}$ (transverse propagation), the above pair of coupled equations reduces to a pair of separate equations, discussed in part I of this paper. In the longitudinal case with $\theta=0$ or $\pi$, the linear combinations $\left(E_{x} \pm i E_{y}\right)$ obey separate equations similar to the one satisfied by the transverse ordinary or $o$-mode. Except for these limiting cases, separate wave equations obeyed by linear combinations of the type $\left(f_{1}(z) E_{x}+f_{2}(z) E_{y}\right)$ cannot be set up for any type of functional dependence of $N$ with $z$. Hence general magneto-ionic propagation is always governed by coupled wave equations.

\section{Nature of the Solutions}

For frequencies used in radio communications $\omega_{H} \ll \omega$, i.e., $\left|z_{0}^{2}\right| \ll l^{2}$, and hence solutions for the region $z^{2} \gg\left|z_{0}^{2}\right|$ are of more practical importance than those for the magneto-ionic core with $z^{2}<\left|z_{0}^{2}\right|$. Changes in the variables from $E_{x}, i E_{y}$, and $z$ to $u_{1}=\xi^{1 / 4}: E_{x}, u_{2}=\xi^{1 / 4} E_{y}$, and $\xi=i K z^{2}$ reduce (3) to the form

$$
\frac{d^{2}}{d \xi^{2}} u_{1,2}+\left[-\frac{1}{4}+\frac{M_{1,2}}{\xi}+\frac{\Lambda_{1,2}}{\xi\left(\xi-\xi_{0}\right)}+\frac{\frac{3}{16}}{\xi^{2}}\right] u_{1,2}=-Q\left[-\frac{1}{4}+\frac{M}{\xi}+\frac{\Lambda}{\xi\left(\xi-\xi_{0}\right)}\right] u_{2,1},
$$

where

$$
\begin{aligned}
M_{1,2} & =\frac{i K}{4}\left[a_{1,2}^{2}+b_{1,2}^{2}-z_{0}^{2}\right], & M & \frac{i K}{4}\left[a_{1}^{2}+l^{2}-z_{0}^{2}\right), \\
\Lambda_{1,2} & =\frac{K^{2}}{4}\left[z_{0}^{2}-a_{1,2}^{2}\right]\left[z_{0}^{2}-b_{1,2}^{2}\right], & \Lambda & =\frac{K^{2}}{4}\left(z_{0}^{2}-a_{1}^{2}\right)\left(z_{0}^{2}-l^{2}\right), \\
\xi_{0} & =i K z_{0}^{2} . & &
\end{aligned}
$$

If $u_{1} \rightarrow u_{1 \infty}, u_{2} \rightarrow u_{2 \infty}$ as $\xi \rightarrow \infty$, the equations obeyed by the asymptotic functions $u_{1 \infty}, u_{2 \infty}$, when the terms of the order of $1 / \xi^{2}$ and smaller are neglected, assume the form

$$
\begin{aligned}
& \frac{d^{4} u_{1 \infty}}{d \xi^{4}}+\left(-\frac{1}{2}+\frac{2 \mu}{\xi}\right) \frac{d^{2} u_{1 \infty}}{d \xi^{2}}+\left[\frac{1-Q^{2}}{16}+\frac{\mu}{2 \xi}\right] u_{1 \infty}=0, \\
& u_{2 \infty}=-\frac{4}{Q}\left[\frac{d^{2} u_{1 \infty}}{d \xi^{2}}+\left(-\frac{1}{4}+\frac{M_{1}}{\xi}\right) u_{1 \infty}\right], \\
& 2 \mu=M_{1}+M_{2} .
\end{aligned}
$$

Solutions of (5) can be expressed as

$$
u_{1 \infty}=\int_{C} e^{\eta \xi} \prod_{j=1}^{4}\left(\eta-\eta_{j}\right)^{\alpha_{j}} d \eta
$$


where

$$
\alpha_{j}=-\left(1+\mu / 2 \eta_{j}\right), \quad \eta_{1,2}= \pm \frac{1}{2} \sqrt{1+Q}, \quad \eta_{3,4}= \pm \frac{1}{2} \sqrt{1-Q}
$$

and the contour $C$ is a combination of four loops, $C_{j}$ 's, $j=1$ to 4 , each round one of the four singularities $\eta_{j}= \pm \sqrt{1 \pm Q}$ of the integrand. Along any ray in the $\eta$-plane, the contribution to (6) from the loop $C_{j}$ can be written as

$$
\begin{aligned}
\left(u_{1 \infty}\right)_{j} & =\int_{C_{j}} e^{\eta \xi}\left(\eta-\eta_{j}\right)^{\alpha_{j}}\left[\prod_{k \neq j}^{1,3}\left(\eta-\eta_{k}\right)^{\alpha} k\right] d \eta \\
& =P_{1 j} e^{\eta_{j} \xi}\left[1+\sum_{p=1}^{\infty}\left(A_{j p} / \xi^{p}\right)\right]
\end{aligned}
$$

where $\boldsymbol{P}_{1 j}$ is the phase factor independent of $\xi$. Evaluation of these phase factors from the asymptotic solutions of the type (6) is necessary for the study of the Stoke's phenomena and the estimation of the observables. The solutions of (4) can now be expressed as

$$
u_{1}=\sum_{j=1}^{4} P_{1 j} e^{\eta_{j} \xi} F_{1 j}(\xi), \quad u_{2}=\sum_{j=1}^{4} P_{2 j} e^{\eta_{j} \xi} F_{2 j}(\xi),
$$

where $\boldsymbol{F}_{1 j}(\xi)$ and $\boldsymbol{F}_{2 j}(\xi)$ are series in descending powers of $\xi$ with unity as the leading term. These series can be evaluated by substituting (6a) in (4) and equating the coefficients of the different powers of $(1 / \xi)$ for each of the four wave terms $\mathrm{e}^{\eta_{j} \xi}$, after $1 /\left(\xi-\xi_{0}\right)$ has been expanded. Comparison of these solutions with those for the transverse modes reveals that the wave factors $\exp$ $\left[+\frac{1}{2} \sqrt{1+Q} \xi\right]$ and $\exp \left[-\frac{1}{2}: \sqrt{1+Q} \xi\right]$ represent respectively the upgoing and downcoming components of the $o$-mode while $\exp \left[\frac{1}{2} \sqrt{1-Q} \xi\right]$ and $\exp \left[-\frac{1}{2} \sqrt{1-Q} \xi\right]$ represent those components of the $e$-mode.

The four observables, viz, $R$, the ratio of amplitude of the downcoming to that of the upgoing wave, $\phi$, their relative phase difference, $\mathscr{R}$, the ratio of the axes of the polarization ellipse of the downcoming wave, and $\Psi$, the tilt of the major axis of that ellipse are related to these solutions as

$$
\begin{aligned}
R e^{-i \phi} & =\left|\frac{P_{13} F_{13}(\xi)}{P_{14} F_{14}(\xi)} \cdot e^{\sqrt{1+Q} \cdot \xi}\right|_{z=-l} \text { for the } o \text {-mode, } \\
& =\left|\frac{P_{11} F_{11}(\xi)}{P_{12} F_{12}(\xi)} \cdot e^{\sqrt{1-Q} \cdot \xi}\right|_{z=-l} \text { for the } e \text {-mode, } \\
R e^{-i \psi} & =\left|\frac{P_{23} F_{23}}{P_{13} F_{13}}\right|_{z=-l} \text { for the downcoming } \boldsymbol{o} \text {-mode, } \\
& =\left|\frac{P_{21} F_{21}}{P_{11} F_{11}}\right|_{z=-l} \text { for the downcoming } e \text {-mode, }
\end{aligned}
$$

$$
\text { and } \Psi=\frac{1}{2} \tan ^{-1}\left[2 \mathcal{R} \sin \psi /\left(1-\mathcal{T}^{2}\right)\right]
$$

Evaluations of these solutions in $(6 a)$ and expressions in $(7,8)$ complete the study of the general magneto-ionic propagation in a parabolic ion layer. Before passing on to the evaluations of the expressions for the observables, the following salient features of the propagation as predicted by the above analysis need to be stressed:

(i) There are only two modes of propagation characterized by the indices $\eta_{1,2}= \pm \frac{1}{2} \sqrt{1+Q}$ and $\eta_{3,4}= \pm \frac{1}{2} \sqrt{1-Q}$, with the two signs of each mode giving the waves traveling in opposite 
directions for that mode. This result holds for any type of functional dependence of $N$ and $\nu$ with $z$. Hence the presence of a third echo (triple-splitting observed in polar latitudes) is ruled out from the general magneto-ionic propagation.

(ii) The complex polarization ratio $e^{-i \psi}$ for the upgoing wave of either mode is not the same as that of the downcoming wave of the same mode. If it were so, suitable linear combinations of the dependent variables would give two decoupled second-order wave equations.

(iii) The sharing of the energy between the two modes is controlled by the parameters of the problem and the propagation angle. This gives rise to a new observable.

\section{Expressions for the Observables}

The nature of the analytic solutions being known, a method based on the basic characteristics of (3) and their solutions (6a) will now be developed for the evaluations of the expressions for the observables.

Writing $i E_{y}=\rho(z) E_{x}$ in (3) and eliminating $E_{x}$ and $i E_{y}$ from the pair of equations by raising the order of differentiation, it can be shown that $\rho(z)$ satisfies the equation,

$$
\begin{aligned}
& 2 \rho \rho^{\prime \prime \prime}-3 \rho^{\prime \prime 2}+4 \rho^{\prime \prime}\left[\rho\left(K_{1}-K_{2}\right)+Q L\left(1-\rho^{2}\right)\right] \\
& \quad-2 \rho^{\prime 2}\left[3 K_{1}-K_{2}-4 \rho Q L\right]-2 \rho^{\prime}\left[\rho\left(K_{1}^{\prime}-K_{2}^{\prime}\right)+Q L^{\prime}\left(1-\rho^{2}\right)\right] \\
& -\left[\left(K_{1}-K_{2}\right) \rho+Q L\left(1-\rho^{2}\right)\right]^{2}=0,
\end{aligned}
$$

where the dashes represent the derivatives with respect to $z$.

Corresponding to each solution $\rho_{j}$ of (9), the components $E_{x}$ and $E_{y}$ of the electric vector satisfy the first-order equations

$$
\frac{d E_{x}}{d z}+\left[\frac{\rho_{j}^{\prime \prime}-\left\{\left(k_{1}-K_{2}\right) \rho_{j}+Q L\left(1-\rho_{j}^{2}\right)\right\}}{2 \rho_{j}^{\prime}}\right] E_{x}=0,
$$

and

$$
\frac{d E_{y}}{d z}+\left[\frac{\rho_{j}^{\prime \prime}-\left\{\left(K_{1}-K_{2}\right) \rho_{j}+Q L\left(1-\rho_{j}^{2}\right)\right\}}{2 \rho_{j}^{\prime}}-\frac{2 \rho_{j}^{\prime}}{\rho_{j}}\right] E_{y}=0 .
$$

Replacing $i E_{y}$ by $\rho E_{x}$, the first of the equations in (3) can be written as

$$
\frac{d^{2} E_{x}}{d z^{2}}+\left[K_{1}-\rho Q L\right] E_{x}=0
$$

Since for parabolic layers, solutions of (3) are symmetrical about $z=0$ and have an essential singularity of grade 2 at $\infty$ together with a pair of regular singularities at $z^{2}=z_{0}^{2}$, the expression $\left(K_{1}-Q \rho L\right)$ in the above equation must fulfill the following conditions:

(i) It must be symmetric about $z=0$;

(ii) It must not contain powers of $z$ higher than the second;

(iii) It must not have any pole other than those at $z^{2}=z_{0}^{2}$.

From the expressions of $K_{1}$ and $L$, it follows immediately that $\rho(z)$ must be of the form

$$
\rho(z)=q+\sum_{1}^{\infty} \frac{\beta_{n}}{\left(z^{2}-z_{0}^{2}\right)^{n}}
$$

Substituting (12) in (9) and equating the coefficients of different powers of $z^{2}$ and $\left(z^{2}-z_{0}^{2}\right)$ 
to zero, it can be shown that

$$
\begin{aligned}
q & = \pm 1, \quad \beta_{1}=h_{2} h_{3} l^{2} \sin ^{2} \theta / 2 \cos \theta=\left(\frac{\omega \omega_{H}}{\omega_{m}^{2}}\right) \cdot l^{2}\left(\frac{\sin ^{2} \theta}{2 \cos \theta}\right) \\
\beta_{2} & =\frac{\beta_{1}^{2}}{2}\left[\left\{\frac{1}{q}-\frac{4 Q}{1-Q^{2}}\right\} \pm \frac{i \sigma}{q}\left\{\sqrt{\left(1-Q^{2}\right)(1+q Q)}\right\}\right], \\
\sigma & =\frac{4(1-i Z)^{-3 / 2}}{\pi h_{1} h_{3}^{2} \sin ^{2} \theta}=\left(\frac{8 c \omega_{m}}{\omega_{H}^{2} l}\right) \cdot \frac{1}{\sin ^{2} \theta},
\end{aligned}
$$

and for all values of $n>2, \beta_{n}$ 's are single-valued.

Since for each root of $q$, there are two roots of $\beta_{2}$ and for each pair of values of $q$ and $\beta_{2}$ all the other $\beta_{n}$ 's are single-valued, (9) has four and only four distinct solutions. Corresponding to each solution of (9), a pair of solutions for $E_{x}$ and $E_{y}$ can be evaluated from (10). The two roots of $q$ characterize the two distinct modes of propagation, while for any of these modes, the two roots of $\beta_{2}$ specify the upgoing and downcoming waves belonging to this mode. The method outlined here is exact and not approximate, though it is not applicable for values of the parameters $h_{3}$ and $\theta$ which make the series (12) divergent. For ionospheric propagation the method is applicable to all latitudes excepting a small belt of $5^{\circ}$ around the equator.

The complex phase factor $R e^{-i \phi}$ between the upgoing and downcoming waves at the bottom of the layer depends on the asymptotic solutions of (11). For such solutions the series (12) can be approximated to $\rho \sim q+\beta_{1} /\left(z^{2}-z_{0}^{2}\right)$, the errors thus introduced being not more than those inherent in all asymptotic expansions. This approximation, however, ignores the subtle difference between the polarization ratios of the upgoing and downcoming waves.

Substituting this asymptotic form of $\rho(z)$ in (11) and simplifying, it can be shown that the general type of magneto-ionic propagation (excluding a small region around $\theta=\pi / 2$ ) is characterized by the wave equation

$$
\frac{d^{2} E_{x}}{d z^{2}}+K_{q}^{2}\left[z^{2}-\frac{M_{q}+\frac{1}{4}}{\left(i K_{q} / 4\right)}+\frac{\left(4 \Lambda / K_{q}^{2}\right)}{\left(z^{2}-z_{0}^{2}\right)}\right] E_{x}=0, \quad E_{y}=-i \rho E_{x}
$$

where

$$
\begin{aligned}
& K_{q}^{2}=4 \pi^{2} h_{1}^{2} /(1-q Q) l^{4}, \quad q= \pm 1 \\
& M_{q}=\frac{i K_{q} l^{2}}{4}\left[1-h_{2}^{2}+q h_{2} h_{3} \cos \theta-\frac{h_{3}^{2} \sin ^{2} \theta}{2(1-q Q)}\right]-\frac{1}{4}, \\
& \Lambda_{q}=\frac{K_{q}^{2} l^{4} h_{3}^{2} \sin ^{2} \theta}{2\left(1-Q^{2}\right)}\left[h_{3}^{2}\left(1+\sin ^{2} \theta\right)-q h_{2} h_{3} \cos \theta-h_{2}^{2}+\frac{q h_{3}^{3} \cos ^{3} \theta}{h_{2}}\right] .
\end{aligned}
$$

Equation (14) being similar to the transverse $e$-mode wave equation discussed in part I, the same procedure may be used for evaluating the phase factor $R e^{-i \phi}$. Carrying out the simplifications and neglecting terms of the order of $h_{3}^{2}$ and smaller, it can be shown that

$$
\begin{gathered}
\left(\frac{c}{\pi l \omega_{m}}\right) \ln \left(\frac{\bar{R}_{q}^{2}}{1-\bar{R}_{q}^{2}}\right)-f_{q}=\frac{1-\left(\frac{\omega^{2}}{\omega_{m}^{2}}+\frac{\omega_{H}^{2} \sin ^{2} \theta}{\omega_{m}^{2}}\right)\left(1-\frac{q \omega_{H} \cos \theta}{\omega}\right)}{\sqrt{1-\frac{q \omega_{H} \cos \theta}{\omega}}}, \\
\chi_{q} \sim \frac{g \nu}{2}\left[\left(1-\frac{f_{q}}{2}\right) \ln \left(\frac{4}{f_{q}}\right)-1\right] /\left[1-q \frac{\omega_{H} \cos \theta}{\omega}\right]^{3 / 2},
\end{gathered}
$$




$$
\begin{aligned}
& \tau_{q} \sim \frac{2 h_{b}}{c}+\frac{\phi_{q}}{\omega}=\frac{2 h_{b}}{c}+2 y_{q}-\pi / \omega, \\
& y_{q} \sim g\left[1-\frac{f_{q}}{2}-\frac{f_{q}}{2} \ln \left(\frac{4}{f_{q}}\right)\right] / \sqrt{\left[1-q \frac{\omega_{H} \cos \theta}{\omega}\right]} \\
& g=\frac{\omega_{m}}{\omega} \frac{l}{c},
\end{aligned}
$$
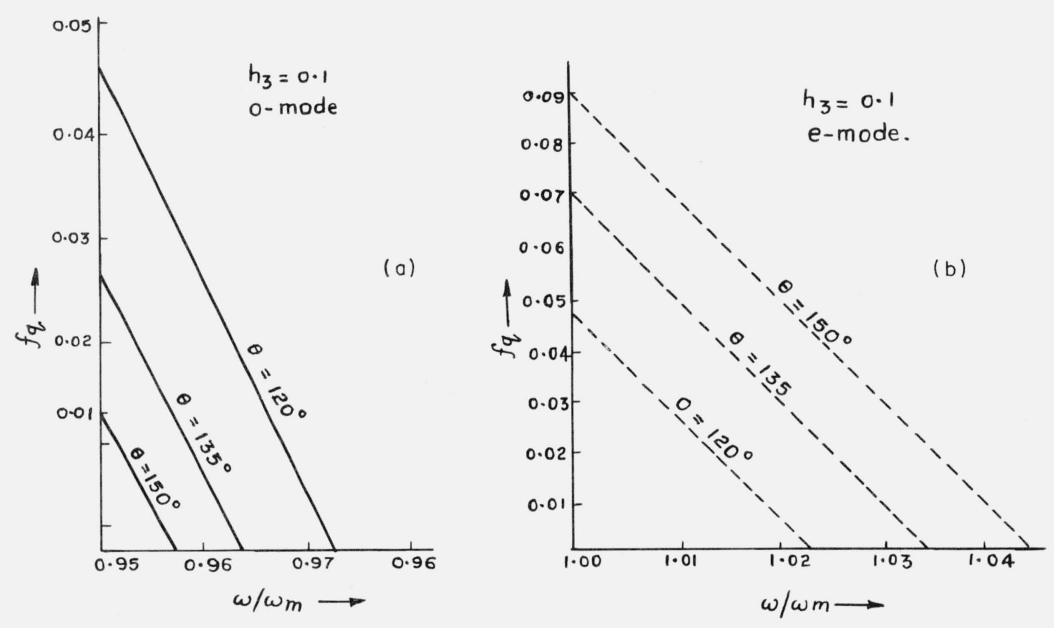

FigURE 1. Reflection coefficient as a function of frequency.
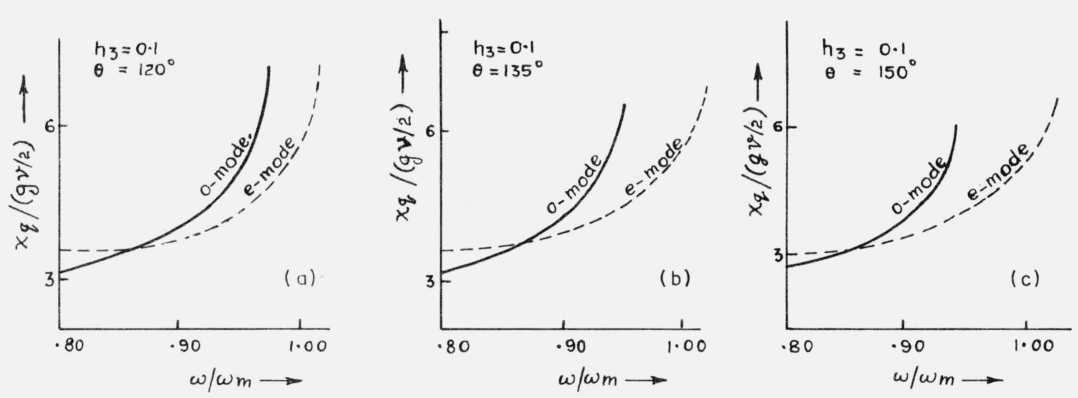

FigURE 2. Absorption coefficient as a function of frequency.
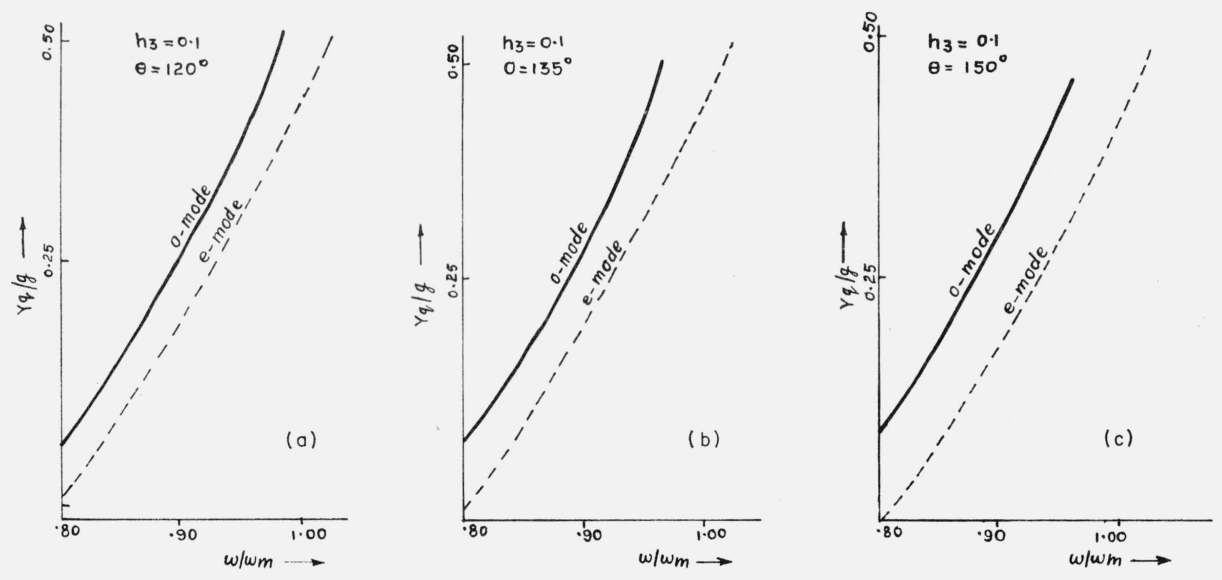

FIGURE 3. Time delay as a function of frequency. 
where $\bar{R}_{q}^{2}$ is the reflection coefficient of the $q$-mode for a friction-free layer, $\chi_{q}$ is its total absorption in the layer and $\tau_{q}$ is the observed time delay in the CRO screen. The $o$-mode is $q=+1$, while the $e$-mode is $q=-1$. Variations of $f_{q}, \chi_{q} /(g \nu / 2)$, and $y_{q} / g$ with $\omega / \omega_{m}$ for $\theta=150^{\circ}, 135^{\circ}$, and $120^{\circ}$ have been plotted in figures 1,2 , and 3 . The $o$-modes are shown by solid curves, and the $e$-modes by dotted curves. The two modes exchange their roles when $\theta$ is replaced by $(\theta-\pi / 2)$.

\section{Polarization of the Downcoming Waves}

The electromagnetic (em) waves inside a magneto-ionic medium are not transverse with respect to the electric vector; hence, it has become customary to study the polarization of the waves with respect to the magnetic vector, which for vertical propagation is always confined to the horizontal plane. Since $\mathbf{E} \cdot \mathbf{H}=0$ even for an inhomogeneous magneto-ionic medium,

$$
H_{x} / H_{y}=-E_{y} / E_{x}=i \rho=R e^{i\left(\frac{\pi}{2}-\psi\right)},
$$

where $\rho$ is given by (12) and (13). Equation (16) shows that the vector whose components are $H_{x}$ and $H_{y}$ traces an ellipse in the $x y$-plane, the ratio of the major to the minor axes being given by $\mathscr{T} R$, whereas the inclination $\Psi$ (tilt angle) of the major axis to the magnetic meridian ( $x z$-plane) is given by

$$
\tan 2 \Psi=\frac{2 \% \sin \psi}{\left(1-R^{2}\right)}
$$

While evaluating the expression for the complex phase factor $R e^{-i \phi}$, retention of terms up to $\beta_{1} /\left(z^{2}-z_{0}^{2}\right)$ in (12) is sufficient to predict results within the limits of experimental accuracy. In the polarization studies, however, terms up to $\beta_{2} /\left(z^{2}-z_{0}^{2}\right)^{2}$ need be retained.

Integration of (10) when $\rho=q+\frac{\beta_{1}}{z^{2}-z_{0}^{2}}+\frac{\beta_{2}}{\left(z^{2}-z_{0}^{2}\right)^{2}}$ gives the dominant term as

$$
E_{x} \sim \frac{z^{-g_{0} z_{0}^{2}} \exp \left[g_{0} z_{0}^{2} / 2\right]}{\sqrt{(d \rho / d z)}}
$$

where

$$
g_{0}=-K^{2} Q\left[2 q\left(z_{0}^{2}-a_{1}^{2}\right)-\frac{\beta_{1}}{2}+\frac{2 q \beta_{2}}{\beta_{1}}\right]
$$

Since $z$ is measured from the tip of the layer, the downcoming wave is given by that root of $\beta_{2}$ for which $\operatorname{Im} g_{0} \leqslant 0$. Hence the limiting polarization $\rho_{l}$ for the downcoming wave is given by

$$
\rho_{l} \sim q+\frac{\beta_{1}}{\left(l^{2}-z_{0}^{2}\right)}+\frac{\frac{1}{2} \beta_{1}^{2}}{\left(l^{2}-z_{0}^{2}\right)^{2}}\left[\left(\frac{1}{q}-\frac{4 Q}{1-Q^{2}}\right)+\frac{i \sigma}{q} \sqrt{\left(1-Q^{2}\right)(1+q Q)}\right] .
$$

Separating the real and imaginary parts of (19) and neglecting terms containing $\nu^{2} / \omega^{2}$ or $\sigma \nu / \omega$, it can be shown that

$$
\begin{aligned}
& \mathscr{T}_{q} \sim\left(q+y \omega_{c}+\frac{1}{2 q} y^{2} \omega_{c}^{2}\right)+\text { terms of the order of } h_{3}^{4}, \\
& \Psi_{q} \sim \frac{-Z y \omega_{c}\left[1+y \omega_{c}\left(1+4 y_{T}^{2}\right) / q\right]}{\mathscr{T}_{q}}-\frac{\omega_{c}^{2} y^{2} \sigma}{\mathscr{R}_{q}} \cdot\left(1+2 y_{T}^{2}\right) \sqrt{\left(1-y_{L}^{2}\right)\left(1+q y_{L}\right)}, \\
& y=\omega_{H} / \omega, \quad y_{L}=y \cos \theta, \quad y_{T}=y \sin \theta, \quad \omega_{c}=\sin ^{2} \theta / 2 \cos \theta .
\end{aligned}
$$

The expression for $\mathscr{R}_{q}$, the ratio of the axes of the polarization ellipse, is the same as that 
deduced by the ray optics, where electron density is assumed to be constant. Expression (21) for the tilt angle $\Psi$ contains two terms, $\Psi_{1 q} \propto \nu$, the collision frequency, and $\Psi_{2 q} \propto \sigma$, i.e. $1 /\left(l \lambda_{m}\right)$.

The second term, which depends on the characteristics $l$ and $\omega_{m}$ of the layer, is absent in the equivalent expression deduced from the ray optics. For ionospheric layers, the second term $\Psi_{2 q}$ is greater than the first term $\Psi_{1 q}$, i.e., for these layers the effect of the nonhomogenity on the tilt angle $\Psi$ is more pronounced than the effect of the collision frequency $\nu$ on it. If $\Psi_{o}$ and $\Psi_{e}$ represent the tilt angles for the $o$ - and $e$-modes respectively, then

$$
\Psi_{0}+\Psi_{e} \sim \frac{\omega_{m} \omega}{\omega_{H}^{2}} \cdot \frac{2 c}{l \cos \theta}
$$

Experimental results on these tilt angles can thus be used to predict the value of $l$, the half-width of the layer.

\section{Conclusions}

The propagation of em waves in a magneto-ionic medium with a parabolic electron distribution has been discussed in detail, and expressions for the observables have been derived. The coupled wave equations for the general station do not give rise to any third echo as expected by Rydbeck [1950]. ${ }^{1} \quad$ For other types of layers, or with collision damping as a function of the vertical height, the nature of the solutions will be different from those given in the text of the paper, though the basic predictions will not be affected to any marked extent. Expressions for the reflection coefficient and the ratio of the axes of the polarization ellipse agree, within limits of experimental errors, with those deduced from the ray optics. The equivalent height-frequency curve for a parabolic layer as deduced from the ray method would be an ellipse. It is not so from the wave optical standpoint. Similarly, the expressions for the tilt angle, as deduced by the two methods, differ radically from each other. The sharing of the energy between the two modes on entering the layer is, as discussed in the body of the paper, determined by the characteristics of the station. This method of evaluating the asymptotic solutions, and thereby the expressions for the observables, can be utilized for any other type of electron layer as well.

\section{Appendix: List of Symbols and Äbbreviations}

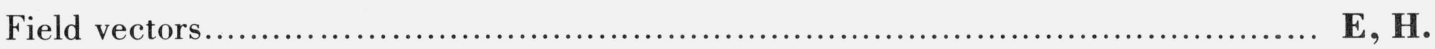

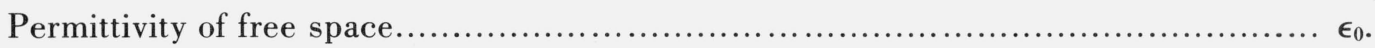

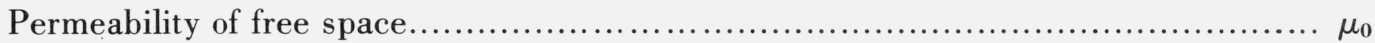

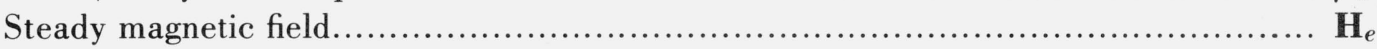

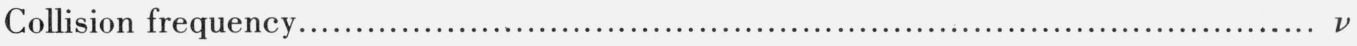

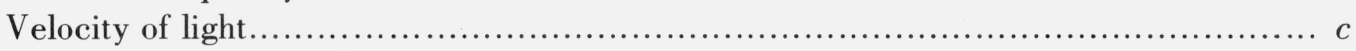

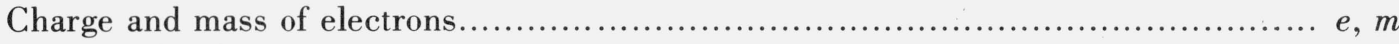

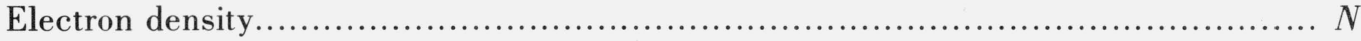

Maximum electron density....................................................... $N_{m}$

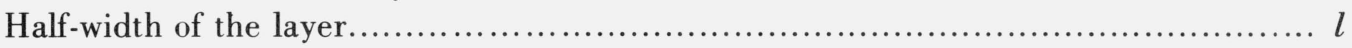

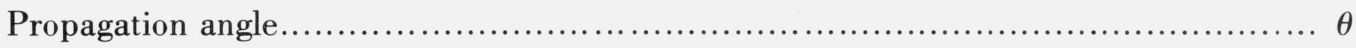

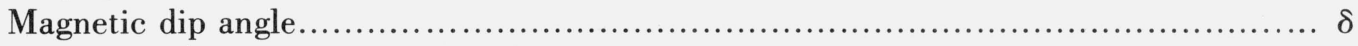

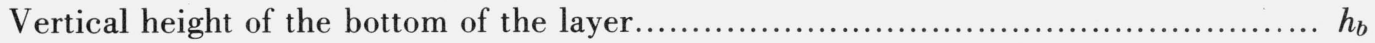

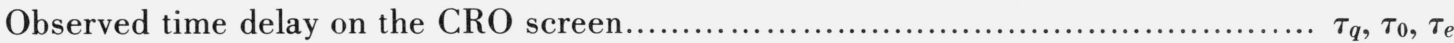

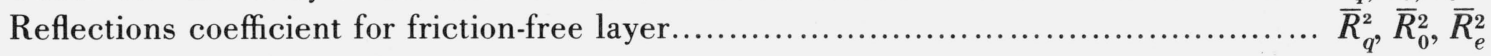

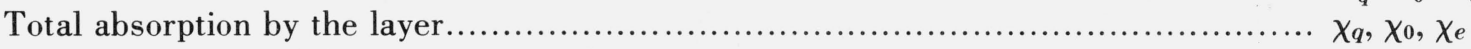

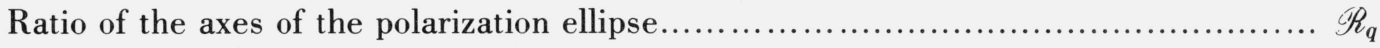

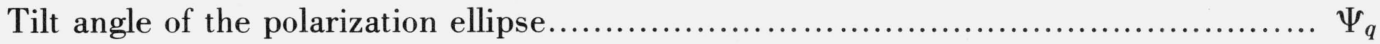

${ }^{1}$ O. E. H. Rydbeck (1950), Natl. Acad. Sci. (India) 4, 329. 
Abbreviations:

$$
\begin{aligned}
& \boldsymbol{\omega}_{H}=e \mu_{0} \mathbf{H}_{e} / m \text {, } \\
& y=\omega_{H} / \omega, \\
& y_{T}=y \sin \theta, \quad y_{L}=y \cos \theta, \\
& \omega_{m}^{2}=N_{m} e^{2} / m \epsilon_{0} \\
& Z=\nu / \omega, \\
& \omega_{c}=\sin ^{2} \theta / 2 \cos \theta, \quad Q=h_{3} \cos \theta / h_{2} \\
& =4 \pi^{2} c^{2} / \lambda_{m}, \\
& h_{1}^{2}=l^{2} /(1-i Z) \lambda_{m}^{2}, \\
& h_{2}^{2}=(1-i Z) \omega^{2} / \omega_{m}^{2}, \\
& h_{3}^{2}=v_{H}^{2} /(1-i Z) \omega_{m}^{2}, \\
& K_{1}=K^{2} \frac{\left(z^{2}-a_{1}^{2}\right)\left(z^{2}-b_{1}^{2}\right)}{\left(z^{2}-z_{0}^{2}\right)}, \\
& K_{2}=K^{2} \frac{\left(z^{2}-a_{2}^{2}\right)\left(z^{2}-b_{2}^{2}\right)}{\left(z^{2}-z_{0}^{2}\right)}, \\
& L=K^{2} \frac{\left(z^{2}-a_{1}^{2}\right)\left(z^{2}-l^{2}\right)}{\left(z^{2}-z_{0}^{2}\right)}, \\
& K^{2}=4 \pi^{2} h_{1}^{2} /\left(1-Q^{2}\right) l^{4}, \\
& z_{0}^{2}=\left[1-h_{2}^{2}+\frac{h_{3}^{2} \sin ^{2} \theta}{1-Q^{2}}\right] l^{2}, \quad g=\frac{\omega_{m}}{\omega} \cdot \frac{l}{c}, \\
& a_{1}^{2}=\left(1-h_{2}^{2}\right) l^{2}, \quad a_{2}^{2}=\left[1-h_{2}^{2}+\frac{h_{3}^{2} \cos ^{2} \theta}{2}+h_{2} h_{3} \sqrt{\sin ^{2} \theta+\frac{Q^{2} \cos ^{2} \theta}{4}}\right] l^{2}, \\
& b_{1}^{2}=\left(1-h_{2}^{2}+h_{3}^{2}\right) l^{2}, \quad b_{2}^{2}=\left[1-h_{2}^{2}+\frac{h_{3}^{2} \cos ^{2} \theta}{2}-h_{2} h_{3} \sqrt{\sin ^{2} \theta+\frac{Q^{2} \cos ^{2} \theta}{4}}\right] l^{2}, \\
& M_{1,2}=\frac{i K}{4}\left(a_{1,2}^{2}+b_{1,2}^{2}-z_{0}^{2}\right), \quad M=\frac{i K}{4}\left[a_{1}^{2}+l^{2}-z_{0}^{2}\right] \\
& \Lambda_{1,2}=\frac{K^{2}}{4}\left(z_{0}^{2}-a_{1,2}^{2}\right)\left(z_{0}^{2}-b_{1,2}^{2}\right), \quad \Lambda=\frac{K^{2}}{4}\left(z_{0}^{2}-a_{1}^{2}\right)\left(z_{0}^{2}-l^{2}\right) . \\
& \xi=i K z^{2}, \quad \xi_{0}=i K z_{0}^{2}, \quad 2 \mu=M_{1}+M_{2}, \\
& \eta_{1,2}= \pm \frac{1}{2} \sqrt{1+Q}, \quad \eta_{3,4}= \pm \frac{1}{2} \sqrt{1-Q}, \quad \alpha_{j}=\left(1+\frac{\mu}{2 \eta_{j}}\right) \text {, } \\
& M_{q}=\frac{i K_{q}}{4} l^{2}\left[1-h_{2}^{2}+q h_{2} h_{3} \cos \theta-\frac{h_{3}^{2} \sin ^{2} \theta}{2(1-q Q)}\right]-\frac{1}{4}, \\
& \Lambda_{q}=\frac{K_{q}^{2} l^{4} h_{3}^{2} \sin ^{2} \theta}{2\left(1-Q^{2}\right)}\left[h_{3}^{2}\left(1+\sin ^{2} \theta\right)-q h_{2} h_{3} \cos \theta-h_{2}^{2}+\frac{q h_{3}^{3} \cos ^{3} \theta}{h_{2}}\right] . \\
& K_{q}^{2}=4 \pi^{2} h_{1}^{2} /(1-q Q) l^{4} \\
& f_{q}=\frac{\left[1-\left(\omega^{2} / \omega_{m}^{2}+\omega_{H}^{2} \sin ^{2} \theta / \omega_{m}^{2}\right)\right]\left[1-q \omega_{H} \cos \theta / \omega\right]}{\sqrt{\left[1-q \omega_{H} \cos \theta / \omega\right]}}, \\
& y_{q}=g\left[1-\frac{f_{q}}{2}+\frac{f_{q}}{2} \ln \left(\frac{4}{f_{q}}\right)\right] / \sqrt{\left[1-q \omega_{H} \cos \theta / \omega\right]}, \\
& \chi_{q}=(g v / 2)\left[\left(1-\frac{f_{q}}{2}\right) \ln \left(\frac{4}{f_{q}}\right)-1\right] /\left[1-q \omega_{H} \cos \theta / \omega\right]^{3 / 2}, \\
& g_{0}=-K^{2} Q\left[2 q\left(z_{0}^{2}-a_{1}^{2}\right)-\frac{\beta_{1}}{2}+\frac{2 q \beta_{2}}{\beta_{1}}\right],
\end{aligned}
$$




$$
\begin{aligned}
\sigma & =\frac{4(1-i Z)^{-3 / 2}}{\pi h_{1} h_{3}^{2} \sin ^{2} \theta}=\left(8 c \omega_{m} / \omega_{H}^{2} \cdot l\right) / \sin ^{2} \theta, \\
\beta_{1} & =h_{2} h_{3} l^{2} \sin ^{2} \theta / 2 \cos \theta=\left(\frac{\omega \omega_{H}}{\omega_{m}^{2}}\right) \frac{l^{2} \sin ^{2} \theta}{2 \cos \theta}, \\
\beta_{2} & =\frac{\beta_{1}^{2}}{2}\left[\left\{\frac{1}{q}-\frac{4 Q}{1-Q^{2}}\right\}+\frac{i \sigma}{q} \sqrt{\left(1-Q^{2}\right)(1+q Q)}\right], \\
\Psi_{1 q} & =-Z y \omega_{c}\left[1+\frac{y \omega_{c}}{q}\left(1+4 y_{T}^{2}\right)\right] / \mathscr{R}_{q}, \\
\Psi_{2 q} & =-\omega_{c}^{2} y^{2} \sigma\left[\left(1+2 y_{T}^{2}\right) \sqrt{\left(1-y_{L}^{2}\right)\left(1+q y_{L}\right)}\right] / R_{q}, \\
\mathscr{R}_{q} & =\left[q+y \omega_{c}+\frac{1}{2 q} y^{2} \omega_{c}^{2}\right] .
\end{aligned}
$$

(Paper 69D9-564) 